\title{
Exploring the Study of Health Quotient Education in Improving Ischemic Cerebral Apoplexy with Hyperuricemia in Zhuang Nationality Youth
}

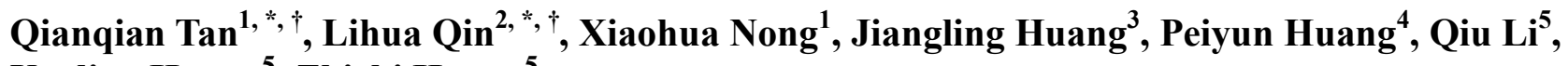 \\ Xuelian Huang ${ }^{5}$, Zhizhi Huang \\ ${ }^{1}$ Departmnt of Nursing, Baise People's Hospital, Southwest Hospital Affiliated to Youjiang Medical College for Nationalities, Baise, China \\ ${ }^{2}$ Department of Osteoarthritis, Guigang Hospital of Integrated Traditional Chinese and Western Medicine, Guigang Red Cross Hospital, \\ Guigang, China \\ ${ }^{3}$ Departmnt of Medical Records Management Office, Baise People's Hospital, Southwest Hospital Affiliated to Youjiang Medical College for \\ Nationalities, Baise, China \\ ${ }^{4}$ Departmnt of Rehabilitation Division, Baise People's Hospital, Southwest Hospital Affiliated to Youjiang Medical College for Nationalities, \\ Baise, China \\ ${ }^{5}$ Departmnt of Neurology, Baise People's Hospital, Southwest Hospital Affiliated to Youjiang Medical College for Nationalities, Baise, China
}

\section{Email address:}

362445564@qq.com (Qianqian Tan),349679983@qq.com (Xiaohua Nong),787209349@qq.com (Lihua Qin),

H6609197@126.com (Jiangling Huang),hpy1580@126.com (Peiyun Huang),836558273@qq.com (QiuLi),

229704433@qq.com (XueLian Huang), zhizhi9900@163.com (Zhizhi Huang)

${ }^{*}$ Corresponding author

$\dagger$ Qianqian Tan and Lihua Qin are co-first authors.

\section{To cite this article:}

Qianqian Tan, Xiaohua Nong, Lihua Qin, Jiangling Huang Peiyun Huang, Qiu Li, Xuelian Huang, Zhizhi Huang. Exploring the Study of Health Quotient Education in Improving Ischemic Cerebral Apoplexy with Hyperuricemia in Zhuang Nationality Youth. American Journal of Nursing Science. Vol. 8, No. 1, 2019, pp. 26-31. doi: 10.11648/j.ajns.20190801.14

Received: December 17, 2018; Accepted: January 15, 2019; Published: February 4, 2019

\begin{abstract}
Objective: Exploring the application of Health quotient idea in improving symptoms of Ischemic Cerebral Apoplexy with Hyperuricemia in the Zhuang nationality youth. Method: 256 patients with Ischemic Cerebral Apoplexy with Hyperuricemia were enrolled in the neurology department of our hospital from January 2016 to August 2018.; According to the randomization method, 256 patients were divided into study group and control group, 128 cases each; the control group was routinely intervened according to the traditional methods, such as: routine care, health education, discharge guidance, continuous intervention after discharge, etc; the study team conducted Health quotient education on the basis of the control group; the health quotient index, healthy behavior, functional exercise compliance, and blood uric acid level which were measured before intervention and intervention after 3 months, and the recurrence rate of Ischemic Cerebral Apoplexy was counted within 1 year after discharge . Results: The indexes of health quotient, health behavior, compliance of functional exercise and serum uric acid level were compared between the two groups before intervention and the result of the difference was not statistically significant $(\mathrm{P}>0.05)$. After three months of intervention, the indexes of health quotient index, health behavior, compliance of functional exercise and blood uric acid levels of the study group were significantly higher than those of the control group $(\mathrm{P}<0.001)$; the recurrence rate of the study group was $0.78 \%$ within 1 year after the intervention $(1 / 128)$ while the recurrence rate of the control group was $12.5 \%$ (16/128); Comparison between the two groups: $\mathrm{x}^{2}=4.4326, \mathrm{P}=0.0353$. The difference was statistically significant. Conclusion: Through the education of the Health quotient idea, it can effectively improve the indicators of health quotient, health behavior, functional exercise compliance and blood uric acid level in patients with Ischemic Cerebral Apoplexy and Hyperuricemia in Zhuang nationality youth and can promote patients to develop healthy ways of life behavior, thus effectively preventing the recurrence of Ischemic Cerebral Apoplexy with Hyperuricemia, and it is worth promoting.
\end{abstract}


Keywords: Health Quotient Idea, Zhuang Nationality Youth, Ischemic Cerebral Apoplexy, Hyperuricemia, Health Quotient Education

\section{Introduction}

Ischemic Cerebral Apoplexy as a common and frequently-occurring disease in the clinic, research shows that with the continuous development of social economy, the trend of Ischemic Cerebral Apoplexy has been transformed from middle-aged to young [1-3]. The main cause of morbidity and recurrence is poor health behavior. Liu Yan et al showed that $83 \%$ of young Ischemic Cerebral Apoplexy patients were from poor health behavior [4]. The risk factors for Ischemic Cerebral Apoplexy in young people are mainly hypertension, hyperuricemia, smoking, and heavy drinking etc. The Zhuang nationality is the most populous minority of the Chinese nation, and the population ranks second among the 56 ethnic groups in China, mainly in the southern part of China. With the continuous deepening of reform and opening up, the economic level of the Zhuang people has been continuously improved, living conditions are getting better and better, lifestyles and diets are constantly changing, and large quantities of high-fat and high-salt foods are consumed. The Zhuang ethnic dwellings have a long history. The eating habits and national culture still maintain unique customs, especially the "big chunks of meat, big bowls of drinking, tobacco and wine are not separated" is the living habit of Zhuang youth. In order to understand the education of the Health Quotient Idea in improving the application in patients with Ischemic Cerebral Apoplexy and Hyperuricemia in young Zhuang nationality, in this study, the Zhuang nationality youth with the symptoms of Ischemic Cerebral Apoplexy and Hyperuricemia in our hospital were educated in the Health Quotient Idea, and the results are reported below.

\section{Data and Methods}

\subsection{Research Object}

All 256 subjects were from young patients with Ischemic Cerebral Apoplexy and Hyperuricemia who were hospitalized in our Neurology Department from January 2016 to August 2018. All patients were male and aged between 28 - 45 years with an average age of (42.24 \pm 9.52$)$ years. The 256 patients were randomly divided into study group and control group, and each group includes 128 patients. The average age of the two groups was $(41.95 \pm 9.96)$ years old and (42.16 \pm 9.71$)$ years old; the occupation, cademic qualification and basic diseases of the two groups were basically the same; there was no significant difference in the general data $(p>0.05)$. All subjects in this study were approved by the Hospital Ethics Management Committee, and the patients or their families signed the informed consent form before enrolled in the study.

\subsection{Subjects Enrolled in Study Selection Criteria and Exclusion Criteria}

1) Matched Criteria: 18 to 45 years old, have a clear mind and a normal thinking; Ischemic Cerebral Apoplexy is the first onset and the diagnosis is consistent with the diagnosis of stroke in the Fourth National Conference on Cerebrovascular Diseases, and confirmed by CT or MRI [5]; Diagnosis of Hyperuricemia: Male blood uric acid $>420 \mu \mathrm{mol} / \mathrm{L}(7.0 \mathrm{mg} / \mathrm{dl})$ [6]; 2) Exclusion Criteria: Mental disorders, dementia, disturbance of consciousness, communication disorders, aphasia, serious complications, loss of follow-up.

\subsection{Research Method}

The control group according to the symptoms of the traditional Ischemic Cerebral Apoplexy combined with Hyperuricemia to take the methods of routine nursing, health education, discharge guidance and continued intervention after discharge. The routine health education of the Ischemic Cerebral Apoplexy and Hyperuricemia mainly includes these such as: diet, medication, psychology, daily life, functional exercise, etc. And the form of education is mainly public health education which includes distributing brochures, pasting departmental publicity windows, organizing group health lectures or focusing on different personality characteristics, habits, psychological conditions and cultural levels of patients to conduct individual health education [7]; In addition, discharge guidance and continuation of nursing intervention were carried on. Such as: using 3D way to guide patients to maintain a good lifestyle and stable mood before discharge, perform functional exercise and medication according to doctor's advice, regular follow-up also be performed, the first telephone follow-up was taken within 1 week after discharge, then the telephone was followed up once every half month for 6 months.

On the basis of the intervention of the control group, the research group implemented the health education of Health Quotient concept; the main measures include: (1) Establishing the Health Quotient Education Committee: the members of the committee are composed of superior doctors, chief physicians, rehabilitation physicians, head nurses, primary nurses and a group of two nurses with rich professional knowledge and strong communication skills. Relevant physicians are responsible for guiding treatment and rehabilitation, solving difficult problems; the head nurses coordinate management; the relevant nurses mainly implement health education on the concept of Health Quotient. (2) Establish patient health records: The health file includes a) General situation: age, gender, ethnicity, education level, occupation, marital status, social status, heredity, daily medication, etc.; b) Physical examination: height, weight, body mass index, blood pressure, limb movement function, etc. c) Living habits: eating habits such as smoking, drinking, nutritional status etc; work and 
rest habits such as sleep, exercise, etc.; d) Medical history: such as current health status, with or without history of familial hereditary diseases. (3) Health Assessment: make a questionnaire on health quotient and health behaviors; Then based on the survey results to explore and analyze the risk factors related to the occurrence of Cerebral Apoplexy and Hyperuricemia in patients; Assess the existing health problems of patients and predict the possible development risks; Identify the risk factors associated with the current disease of the patient, and solicit the needs of health education from patients to make the intervention plan of Health Quotient concept. (4) The interventions of health education of Health Quotient concept. Based on the concept of health quotient and the health behavior problems of patients to combine with different risk factors and possible development trends, patients are given individualized health quotient concepts and health behavior education. Educational form: develop and distribute health education publicity materials according to different needs of patients, one-on-one demonstration teaching, bedside guidance, guidance and answering questions through chat software platform, and continuing health education after discharge.

\subsection{Observation Index}

The following relevant indicators were evaluated before intervention and after 3 months of intervention: (1) Health Quotient Index evaluation: Using the Health Quotient questionnaire from Huazhen Xie [8] which covers five dimensions as below: mental state, self-care, lifestyle, life skills, and health knowledge. Each dimension consists of 4 factors, each of factors contains 5 terms, each of terms has 7 options; The 7 options of terms means never, completely disagree, disagree, basically disagree, basic consent, consent, complete consent, etc. The assignments are 0 to 6 points respectively. The higher the total score, the higher the health quotient, and indicating that the health status is better. The health quotient index is divided into: $<3$ points are divided into health quotient is not good, and important intervention must be carried out immediately; 3 to 5 point are divided into vigilant health quotient, and it is necessary to practice hard to improve health; 6 to 8 points are divided into ideal health quotient, and means that patient has been practicing some methods to maintain health, but which can be done better; 9 to 10 points are divided into the best health quotient, and patient has fully recognized the importance of health quotient to health and applies health knowledge to real life. (2) Health behavior assessment: As assessed by the Health Promotional Lifestyle Rating Scale II (HPIP) [9], the scale contains six dimensions as below : health responsibility, self-fulfillment, exercise, nutrition, interpersonal relationship, and pressure management; The scale has a total of 52 items, and adopt the methods of 4-level rating which is represented as below: 1 point means "never", 2 points means "sometimes", 3 points means "frequently", 4 points means "always", and the full score is 208 points. The higher the score, the better the health behavior. Evaluating grade classification of health behavior: 52 to 91 is divided into poor, 92 to 131 is divided into general,
132 to 171 is divided into good, and 172 to 208 is classified as excellent. (3) Patient functional exercise compliance: Beilei Lin etc. [10] compiled a functional exercise compliance scale for cerebral apoplexy patients. The scale includes physical exercise compliance, exercise effect monitoring compliance and active seeking advice compliance. The scale has 3 dimensions and 20 terms, while each clause adopts 1 to 4 grades which is represented as below : 1 point means that it can not be done at all, 2 points means that it can be done occasionally, 3 points means that it is basic do it, 4 points means that it can be completely done, and the higher the score, the better the compliance; (4) Determination of blood uric acid level: $3 \mathrm{ml}$ of venous blood was collected on an empty stomach in the morning, and be sent to the laboratory for determination of serum uric acid (uric acid oxidase method). (5) Followed up the recurrence rate of ischemic cerebral apoplexy: All patients were followed up for 1 year to count the recurrence rate of ischemic cerebral apoplexy. Recurrence criteria for ischemic cerebral apoplexy [11]: new signs and signs of neurological deficits appear, and new ischemic lesions confirmed by CT or MRI.

\subsection{Statistical Processing}

The data were statistically analyzed using SPSS19.0 software. The health quotient index, health behavior evaluation and health exercise compliance of the two groups which were expressed by $x \pm s$, using t test for statistics, the difference was statistically significant at $\mathrm{p}<0.05$.

\section{Result}

Among the 256 observed subjects, comparison of the dimensions of the two groups of health quotients before intervention, the difference was not statistically significant $(\mathrm{P}$ $>0.05$ ), After 3 months of intervention, the health quotients index of the study group was significantly higher than that of the control group, and the difference was statistically significant ( $\mathrm{P}<0.001)$, see Table 1 , Table 2. There was no significant difference between the two groups in different dimensions of health behavior before intervention $(\mathrm{P}>0.05)$, Three months after intervention, all dimensions of health behavior in study group were significantly higher than those in control group $(\mathrm{P}<0.001)$, as shown in tables 3 , Table 4 . The compliance of functional exercise was compared between the two groups of patients before intervention and intervention after 3 months: Before intervention, there were no significant differences between the two groups in three dimensions of physical exercise compliance, exercise effect monitoring compliance and proactively seeking advice compliance $(\mathrm{P}>$ $0.05)$; three months after intervention, the compliance of the three dimensions in the study group was significantly higher than that in the control group, and the difference was not statistically significant $(\mathrm{P}<0.001)$, as shown in Table 5. The serum uric acid levels were compared between the two groups before intervention and intervention after 3 months: There was no significant difference in serum uric acid level between the two groups before intervention $(\mathrm{P}>0.05)$; after 
intervention, the level of serum uric acid in the study group was significantly lower than that in the control group and the difference was not statistically significant $(\mathrm{P}<0.001)$. See table 6 Comparison of recurrent rate of Ischemic Cerebral Apoplexy between two groups: the recurrence rate was $0.78 \%$
$(1 / 128)$ in the study group and $12.5 \%(16 / 128)$ in the control group within one year after intervention; comparison between the two groups $\mathrm{x} 2=4.4326, \mathrm{P}=0.0353$, the difference is statistically significant.

Table 1. Comparison of the health quotient index between the two groups before intervention and intervention for 3 months ( $n, \bar{x} \pm s$, score).

\begin{tabular}{|c|c|c|c|c|c|c|}
\hline \multirow{2}{*}{ Groups } & \multirow{2}{*}{ Number of cases } & \multicolumn{5}{|c|}{ Before intervention } \\
\hline & & Self health care & Health knowledge & Lifeway & Mental state & Life skills \\
\hline Control Group & 128 & $2.93 \pm 0.61$ & $2.86 \pm 0.51$ & $2.85 \pm 0.58$ & $2.91 \pm 0.58$ & $3.26 \pm 0.71$ \\
\hline Study Group & 128 & $2.90 \pm 0.63$ & $2.82 \pm 0.52$ & $2.83 \pm 0.55$ & $2.92 \pm 0.61$ & $3.23 \pm 0.73$ \\
\hline $\mathrm{t}$ value & - & 0.3870 & 0.6213 & 0.2831 & 0.1344 & 0.3333 \\
\hline$P$ value & - & 0.3495 & 0.2675 & 0.3887 & 0.4466 & 0.3696 \\
\hline
\end{tabular}

Table 2. Comparison of the health quotient index between the two groups before intervention and intervention for 3 months ( $n=50$, score) (continued).

\begin{tabular}{llllll}
\hline \multirow{2}{*}{ Groups } & \multirow{2}{*}{ Number of cases } & After intervention & & & \\
\cline { 3 - 6 } & & Self health care & Health knowledge & Lifeway & Mental state \\
\hline Control Group & 128 & $6.23 \pm 0.77$ & $6.11 \pm 0.64$ & $6.41 \pm 0.75$ & $6.23 \pm 0.68$ \\
Study Group & 128 & $7.92 \pm 0.71$ & $7.71 \pm 0.68$ & $8.16 \pm 0.78$ & $7.32 \pm 0.63$ \\
t value & - & 18.2553 & 19.3850 & 18.2971 & $7.96 \pm 0.66$ \\
P value & - & 0.0000 & 0.0000 & 0.0000 & 13.3033 \\
\hline
\end{tabular}

Table 3. Comparison of health behaviors between the two groups before intervention and intervention for $3 \mathrm{months}$ ( $n$, $\bar{x} \pm s$, score).

\begin{tabular}{|c|c|c|c|c|c|c|c|c|}
\hline \multirow[b]{2}{*}{ Groups } & \multirow{2}{*}{$\begin{array}{l}\text { Number } \\
\text { of cases }\end{array}$} & \multicolumn{6}{|c|}{ Before intervention } & \multirow{2}{*}{$\begin{array}{l}\text { Total score of } \\
\text { health behavior }\end{array}$} \\
\hline & & Self-fulfilment & $\begin{array}{l}\text { Health } \\
\text { responsibility }\end{array}$ & $\begin{array}{l}\text { Stress } \\
\text { management }\end{array}$ & $\begin{array}{l}\text { Interpersonal } \\
\text { relationship }\end{array}$ & Exercise & Nutrition & \\
\hline Control Group & 128 & $18.65 \pm 2.93$ & $17.12 \pm 2.87$ & $18.76 \pm 2.71$ & $23.32 \pm 2.82$ & $14.63 \pm 2.85$ & $18.95 \pm 2.68$ & $111.43 \pm 2.81$ \\
\hline Study Group & 128 & $18.81 \pm 2.84$ & $17.33 \pm 2.96$ & $18.82 \pm 2.69$ & $23.49 \pm 2.88$ & $14.19 \pm 2.97$ & $19.02 \pm 2.71$ & $111.66 \pm 2.84$ \\
\hline $\mathrm{t}$ value & - & 0.4436 & 0.2744 & 0.1778 & 0.4772 & 1.2094 & 0.2078 & 0.6513 \\
\hline$P$ value & - & 0.3288 & 0.3920 & 0.4295 & 0.3168 & 0.1138 & 0.4178 & 0.2577 \\
\hline
\end{tabular}

Table 4. Comparison of health behaviors between the two groups before intervention and intervention for 3 months ( $n, \bar{x} \pm s$, score) (continued).

\begin{tabular}{|c|c|c|c|c|c|c|c|c|}
\hline \multirow[b]{2}{*}{ Groups } & \multirow{2}{*}{$\begin{array}{l}\text { Number of } \\
\text { cases }\end{array}$} & \multicolumn{6}{|c|}{ After intervention } & \multirow{2}{*}{$\begin{array}{l}\text { Total score of } \\
\text { health behavior }\end{array}$} \\
\hline & & Self-fulfilment & $\begin{array}{l}\text { Health } \\
\text { responsibility }\end{array}$ & $\begin{array}{l}\text { Stress } \\
\text { management }\end{array}$ & $\begin{array}{l}\text { Interpersonal } \\
\text { relationship }\end{array}$ & Exercise & Nutrition & \\
\hline Control Group & 128 & $24.18 \pm 3.42$ & $23.24 \pm 3.01$ & $21.96 \pm 2.87$ & $23.96 \pm 2.53$ & $18.97 \pm 2.75$ & $24.78 \pm 3.25$ & $137.09 \pm 2.97$ \\
\hline Study Group & 128 & $28.85 \pm 4.03$ & $27.61 \pm 3.36$ & $27.63 \pm 3.45$ & $28.37 \pm 3.09$ & $22.34 \pm 2.81$ & $29.69 \pm 3.76$ & $164.49 \pm 3.42$ \\
\hline $\mathrm{t}$ value & - & 9.9961 & 10.9599 & 14.2944 & 12.4933 & 9.6973 & 11.1773 & 68.4377 \\
\hline$P$ value & - & 0.0000 & 0.0000 & 0.0000 & 0.0000 & 0.0000 & 0.0000 & 0.0000 \\
\hline
\end{tabular}

Table 5. Comparison of functional exercise compliance between the two groups before intervention and intervention for 3 months (n, $\bar{x} \pm s$, score ).

\begin{tabular}{|c|c|c|c|c|c|c|c|}
\hline \multirow[b]{2}{*}{ Groups } & \multirow[b]{2}{*}{$\begin{array}{l}\text { Number } \\
\text { of cases }\end{array}$} & \multicolumn{3}{|c|}{ Before intervention } & \multicolumn{3}{|l|}{ After intervention } \\
\hline & & $\begin{array}{l}\text { Physical exercise } \\
\text { compliance }\end{array}$ & $\begin{array}{l}\text { Exercise effect } \\
\text { monitoring } \\
\text { compliance }\end{array}$ & $\begin{array}{l}\text { Proactively } \\
\text { seeking advice } \\
\text { compliance }\end{array}$ & $\begin{array}{l}\text { Physical exercise } \\
\text { compliance }\end{array}$ & $\begin{array}{l}\text { Exercise effect } \\
\text { monitoring } \\
\text { compliance }\end{array}$ & $\begin{array}{l}\text { Proactively } \\
\text { seeking advice } \\
\text { compliance } \\
\end{array}$ \\
\hline $\begin{array}{l}\text { Control } \\
\text { Group }\end{array}$ & 128 & $21.92 \pm 2.89$ & $7.06 \pm 1.45$ & $5.62 \pm 1.68$ & $47.28 \pm 4.22$ & $22.68 \pm 3.36$ & $23.78 \pm 3.15$ \\
\hline Study Group & 128 & $21.36 \pm 3.02$ & $6.98 \pm 1.28$ & $5.49 \pm 1.34$ & $66.34 \pm 4.52$ & $30.21 \pm 3.56$ & $35.62 \pm 3.87$ \\
\hline $\mathrm{t}$ value & - & 1.5157 & 0.4680 & 0.6844 & 34.8719 & 17.4031 & 26.8449 \\
\hline
\end{tabular}

Table 6. Comparison of serum uric acid levels before intervention and intervention for 3 months $(\bar{x} \pm s, \mu m o l / L)$.

\begin{tabular}{llll}
\hline Groups & Number of cases & Before intervention & After intervention \\
\hline Control Group & 128 & $491.08 \pm 11.72$ & $427.18 \pm 12.04$ \\
Study Group & 128 & $489.93 \pm 12.13$ & $392.05 \pm 11.87$ \\
t value & - & 0.7714 & 23.5076 \\
P value & - & 0.2206 & 0.0000 \\
\hline
\end{tabular}




\section{Discussion}

Health Quotient is the quotient of health, which refers to the health consciousness, health knowledge and health ability that individuals have and should have [8]. Health quotient is extremely important in the self-management of chronically ill patients [12-13]. Healthy behavior refers to a series of behavioral activities taken by individuals to maintain or promote their health to achieve self-fulfilment or satisfaction [9]. The better the patient's level of healthy behavior, the higher the quality of life. Ischemic cerebral apoplexy has the characteristics of "four highs" which refers to high incidence, high recurrence rate, high disability rate and high mortality. Hyperuricemia (HUA) is a disease caused by abnormal increase of serum uric acid concentration which is caused by purine metabolic disorder; the healthy behavior of patients with Ischemic Cerebral Apoplexy and Hyperuricemia is closely related to the recurrence of the disease [14]. Patients with Ischemic Cerebral Apoplexy are often accompanied by hyperuricemia [15]. If Ischemic Cerebral Apoplexy patients with HUA can not be corrected in time, their condition will develop further, which may lead to complications such as depression [16-18]. Ischemic Cerebral Apoplexy in youth refers to Ischemic Cerebral Apoplexy at the age of 18-45 years [19]. The main reason for the Ischemic Cerebral Apoplexy in the Zhuang youth is that the people in this age group are in a period of hard work and most of the time they are busy with the pressure of work and family life, and there is not enough time and energy for exercise. At the same time, the health awareness is weak, and the main entertainment mode in the spare time is online, drinking, etc., and they don't pay enough attention to their own health. Foreign scholars Gordon etc. [17] have shown that exercise is an independent risk factor for Cerebral Apoplexy, and long-term exercise can prevent and reduce the occurrence and recurrence of Cerebral Apoplexy. The health behavior of patients with Ischemic Cerebral Apoplexy in Zhuang youth is poor, so their health awareness and healthy lifestyle need to be improved. Health care workers should strengthen health education on healthy behavior and moderate physical exercise of young patients with Ischemic Cerebral Apoplexy, and guide patients to make appropriate plans for physical exercise and rehabilitation exercise, so as to enhance patients' understanding of healthy behavior. To effectively control the risk factors of the occurrence, development and recurrence of Ischemic Cerebral Apoplexy. Early intervention and multiple forms of health behavior education should be adopted for young Zhuang patients with Ischemic Cerebral Apoplexy and Hyperuricemia to enhance the health behavior consciousness of the patients, so as to urge the patients to change the bad lifestyle of "big chunks of meat, big bowls of drinking, excessive smoking"and "gather to drink "in spare time, etc.; consciously adopt healthy behavior to reduce or eliminate the risk factors of occurrence, recurrence, or aggravation of Ischemic Cerebral Apoplexy disease; which has a great significance to prevent and control the incidence and recurrence rate of Ischemic Cerebral Apoplexy in Zhuang youth [22].
The results showed that there was no significant difference in health quotient index between the two groups before intervention $(\mathrm{P}>0.05)$; after 3 months intervention, the health quotient index of the study group was significantly higher than that of the control group, the difference was statistically significant $(\mathrm{P}<0.001)$. There was no significant difference in each dimension of health behavior between the two groups before intervention $(\mathrm{P}>0.05)$; after 3 months intervention, all dimensions of health behavior in study group were significantly higher than those in control group and the difference is statistically significant $(\mathrm{P}<0.001)$. Comparison of functional exercise compliance between the two groups, before the intervention, there was no statistically significant difference between the two groups in terms of physical exercise compliance, exercise monitoring compliance, and active seeking advice; the differences in compliance with the three dimensions were not statistically significant $(\mathrm{P}>0.05)$; after 3 months intervention, the compliance of the three dimensions of the study group was significantly higher than that of the control group, and the difference was statistically significant $(\mathrm{P}<0.001)$. There was no significant difference in blood uric acid levels between the two groups before the intervention ( $\mathrm{P}>0.05)$; after 3 months intervention, the blood uric acid level of the study group was significantly lower than that of the control group, and the difference was statistically significant $(\mathrm{P}<0.001)$. Comparison of recurrence rates between two groups of ischemic cerebral apoplexy: the recurrence rate of the study group was $0.78 \%(1 / 128)$ within 1 year after the intervention; the recurrence rate of the control group was $12.5 \%(16 / 128)$; comparison between the two groups $\mathrm{x} 2=4.4326, \mathrm{P}=0.0353$, the difference was statistically significant. This indicated that the education of health quotient concept had better clinical effect in improving Ischemic Cerebral Apoplexy with Hyperuricemia in young Zhuang nationality.

\section{Conclusion}

The health education model of the health quotient concept emphasizes the observation of the whole body and mind of the patient and the role of the social family in the recovery process of the patient. The results show that the health education model of the health quotient concept, which can significantly improve the health quotient of Ischemic Cerebral Apoplexy patients with Hyperuricemia, and improve the cognition of secondary prevention of Ischemic Cerebral Apoplexy, while improve the compliance of functional exercise, and promote the establishment of healthy lifestyle. Therefore, it has good social and practical significance to promote rehabilitation and prevent the recurrence of Ischemic Cerebral Apoplexy.

\section{Limitations of Research}

This study mainly observed the young people of Zhuang nationality, and other nationalities have not been studied, so it has some limitations. In addition, during the follow-up of 
patients, there may be individual differences in the duration of follow-up because the patients live in different places.

\section{Acknowledgements}

The project got the strong support of the hospital functional departments and the help of colleagues so that can be established, here to their support and help to express sincere gratitude! Wish them good health, smooth work and all the best!

\section{Fund Projects}

Scientific Research and Technology Development Project in Baise City of Guangxi (NO.: Encyclopedia 20170502)

\section{References}

[1] Yingying Liu, Youze Han, Ye Wang.Current status of research on etiology and risk factors of ischemic cerebral apoplexy in young people [J]. Stroke and Neurological Diseases, 2017, 24 (4): $371-374$.

[2] Tingting Sun, Peilan Zhang. Research progress in the etiology and pathogenesis of ischemic cerebral apoplexy in young people [J]. Chinese Journal of Practical Nervous Diseases, 2018, 21 (4): 447-449.

[3] Yue Zheng, Lin Yi, Zhuolin Yin, et al. Etiological Analysis of 268 cases of Young Stroke [J]. Chinese Journal of Cerebrovascular Disease, 2011, 5 (2): 116-121

[4] Yan Liu, Yi Jin, Yue Zhao. Analysis of health behaviors and influencing factors in young stroke patients [J]. Chinese Journal of Nursing, 2012, 47 (1): 58-61.

[5] Chinese Medical Association Neuroscience Society.Various types of diagnosis of cerebrovascular diseases $[\mathrm{J}]$. Chinese Journal of Neurology, 1996, 29 (6): 370.

[6] Dayi Hu, Rongjing Ding.Asymptomatic hyperuricemia with cardiovascular disease diagnosis and treatment recommendations Chinese expert consensus [J]. Chinese General Practice, 2010, 13 (11): 1145-1149.

[7] Chunying Liu.The effect of continuing nursing intervention on improving self-care ability and behavior of patients with hypertension [J]. Qilu Nursing Magazine, 2015: 20 (05): 4-6.

[8] Huazhen Xie. Health Quotient HQ [M]. Beijing: China Society Press, 2002: 1-30.

[9] Yajuan Yang, Cailian Fei, Yao Jing. Effects of applying protection motivation theory on the health behavior of first-time young stroke patients [J]. Journal of Nursing of Chinese Peoplel's Liberation Army, 2017, 4 (13): 1-5.
[10] Beilei Lin, Zhenxiang Zhang, Yumei Sun, et al. Compilation and reliability and validity test of functional exercise compliance scale in community stroke patients [J]. Chinese Journal of Rehabilitation Medicine, 2013, 28 (6): 574-578.

[11] Chinese Medical Association Neurology Branch. Chinese Medical Association Neurology Branch Cerebrovascular Disease Group. China's ischemic stroke and transient ischemic attack secondary prevention guidelines 2014 [J]. Chinese Journal of Neurology, 2015, 48 (4): 258-273.

[12] Yujie Zhong, you Wu, Yang Li, et al. Health Quotient Theory: a New Perspective of Community Hypertension Health Management [J]. Chinese Journal of Gerontology, 2015, 35 (4): 1120-1121.

[13] Qingwei Yan, Xiaoming Wu, Jianfang Gong, et al. Discussion on the effect of human health intervention in the management of chronic diseases [J]. Management Observation, 2017: 31 (11): 76-7 7, 80 .

[14] Ping Yu, Liufu Cui, Huijing Shi, et al.The relationship between ideal cardiovascular health behaviors and factors and blood uric acid levels [J]. Chinese Journal of Hypertension, 2015, 23 (8): 749-754.

[15] Fanyan Meng, Tao Gu, YajunWu, et al.Study on the effect of uric acid control therapy on stroke patients with hyperuricemia [J]. Contemporary Medicine Journal, 2016, 14 (2): 138-140 .

[16] Jie Yuan, Yueying Lu, Xin Guo, et al. Predictive value of serum uric acid level in post-acute post-stroke depression [J]. Chinese Journal of General Practice, 2018, 21 (16): 1974-1979.

[17] Wei Liu, Dongjie Li, Liang Zhao . Research the relationship between serum uric acid level and ischemic stroke in young people [J]. Chinese Journal of Clinical Medicine, 2017, 37 (5): 31-33.

[18] Mei Wang, Yuqiang Song.Research progress in the relationship between uric acid and cerebral infarction [J]. Journal of Stroke and Nervous Diseases, 2016, 23 (5): 373-376.

[19] Linmin Huang, Anqi Xu, Xiaotiao Cai, et al. Qualitative study of 12 cases of hospitalized real-life experience in middle-aged and young-age strokes [J]. China Rural Medicine, 2018, 25 (9): 69-70.

[20] Jianping Jia, Shengdi Chen, Neurology [M] Beijing: People's Health Publishing House, 2013: 175-188.

[21] Gordon NF, Gulanic M, Costa F, et a1. Physical activity and exercise recommendations for stroke survivors: an Americn Heart Association scientific statement [J]. Circulation, 2004, 109 (16): 2031-2041.

[22] Lihong Wan, Miaomiao Mo, Xiaopei Zhang, et al.Construction and evaluation of comprehensive behavioral reminder system for discharge of ischemic stroke patients [J]. Chinese General Practice, 2017, 20 (28): 3548-3553. 